\title{
La alternativa socialista
}

El colapso de los regímenes socialistas de la ex Unión soviética y el este de Europa y el aparente fracaso económico de sus economías centralizadas, una vez alcanzado un cierto nivel de desarrollo material, han llevado a levantar rápidamente el acta de defunción del socialismo y a declarar que no hay alternativa al sistema capitalista. La rotunda claridad con que el capitalismo ha expresado en los últimos 50 años, combinando la estrangulación económica con la guerra de alta tecnología y baja intensidad, que no permitirá el más leve desafío, y la sistematicidad con que ha sido aplastada la gran ola de revoluciones armadas más o menos exitosas en Asia, Africa, y América Latina, no han dejado mucho lugar para la duda. El hecho de que muchos asalariados ahora tengan algo más que perder que sus cadenas y la inviabilidad misma de muchas estrategias revolucionarias clásicas al desaparecer el bloque del este parecerían acabar de cerrar todo resquicio para un cambio del sistema a corto y medio plazo. Hay que reconocer que la gran contraofensiva desencadenada en la década pasada por las fuerzas del capital contra la proliferación de movimientos revolucionarios en el tercer mundo triunf6. Nada tiene de extraño que buena parte del pensamiento teóricamente atado a la clase trabajadora y a movimientos antiimperialistas se encuentre en crisis y que muchos de los sobrevivientes de los antiguos movimientos revolucionarios estén deprimidos o desorientados. Los que más se benefician del sistema pueden dormir ahora mucho más tranquilos. Con todo, no podemos decir que entre los cientos de millones de pobres del tercer mundo la esperanza haya desaparecido por completo, si bien una parte de la izquierda, en aras del realismo, se ha apresurado a firmar el acta de defunción, haciendo contrición de su pasado y re- conociendo que lo único que queda en agenda es mejorar los peores aspectos del sistema capitalista existente, hay otra izquierda, a veces no nominal, que lucha por múltiples causas en su quehacer cotidiano, y que, por más oscura que encuentre cualquier alternativa al sistema, es proclive a buscarla.

En este panorama creo que la lectura de un libro como el de David Schweickart, puede provocar un debate y reflexión a todas luces necesario: Más allá de la crítica al sistema, ¿hacia donde queremos empujar el mundo y cómo? Conformarse con mostrar que el capitalismo, con todos sus innegables logros, es, en su origen, en sus operaciones diarias y en su cuerpo teórico, un sistema económico intrínsecamente injusto y enmudecer ante la lacónica pregunta de sus defensores: $i$ muy bien, tienes razón, pero cuál es tu alternativa? Es una concesión que David Schweickart no está dispuesto a hacer. Marx, ciertamente, reflexionó muy poco sobre instituciones alternativas, concentrándose en la crítica del presente y en una teoría histórica sobre el agente de cambio. Probablemente confiaba en que la clase llamada a acceder al poder construiría con su victoria unas instituciones alternativas apropiadas. La crítica incuestionable de Marx al socialismo utópico no es que pensadores como Owens, Fourier o Saint Simon se dedicaran a pensar modelos alternativos, sino que éstos creyeran que llegarían a darse a través de la argumentación ética y de los experimentos comunales divorciados de la lucha política. Hoy es evidente que negarse a pensar una alternativa es más una rémora que una ventaja para articular fuerzas de cambio. Primero, porque en este siglo ha habido muchos experimentos socialistas que no sólo han sido un fracaso, sino una auténtica barbarie $y$, por 
tanto, nadie puede creer ya que la abolición de la propiedad privada de los medios de producción producirá automáticamente una sociedad justa, ni puede comprometerse sin más en esta lucha. Segundo, porque en nuestra época se han hecho muchos experimentos: fascismo, socialismo planificado centralmente, capitalismo planificado, capitalismo con estado mínimo, etc., y no necesariamente todo cambio mejora el sistema capitalista. En la actualidad, no podemos comprometernos ciegamente en ninguna causa sin discutir sobre lo que funciona y lo que no. Tercero, porque si bien en el primer mundo el capitalismo ha triunfado completamente $y$

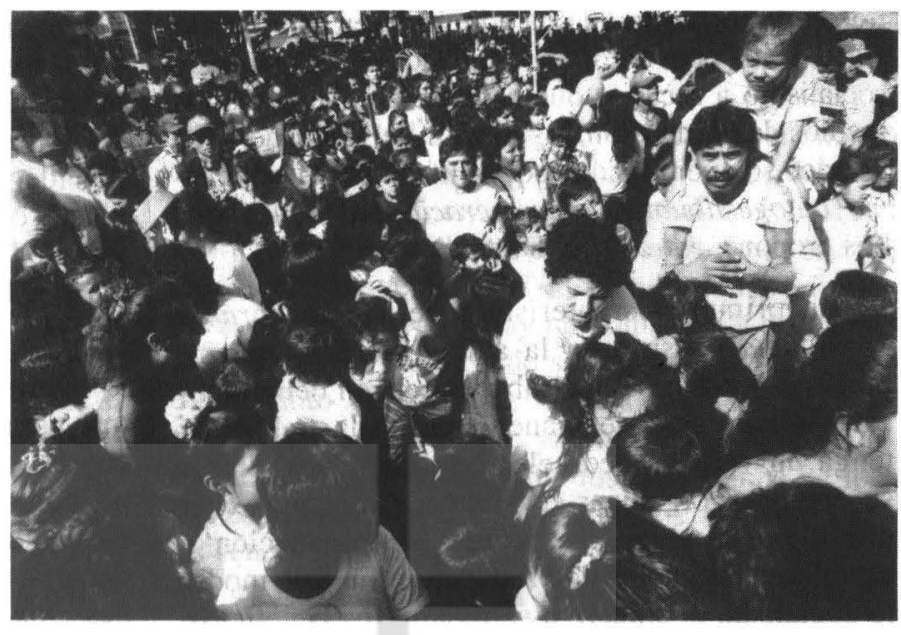

do libre (los precios son determinados por la oferta y la demanda con mayor o menor interferencia del gobierno) y la consideración de la fuerza de trabajo como una mercancía más (la fuerza de trabajo vende su capacidad a aquellos que pueden proveer un lugar de trabajo). Simplificando mucho puede hablarse de un capitalismo en estado puro o capitalismo liberal, cuyo ideal es que la economía esté lo mas alejada posible de la intervención del gobierno y que, después de la revolución conservadora de Reagan, suele identificarse con el conservadurismo o también con el neoliberalismo: privatización, recorte de los servicios sociales, mayor liberalización del mercado y un capitalismo keynesiano o socialdemócrata que considera necesaria la intervención del gobierno para distribuir la riqueza y orientar racionalmente el crecimiento'. Por socialismo entendemos cualquier sistema económico que, más allá de toda reforma, altere alguna de las propiedades esenciales del sistema capitalista anteriormente mencionadas. Fundamentalmente se pueden distinguir aquí, con todas sus variantes, dos modelos: el socialismo de Estado que,

1. Aquí hay que andarse con tiento con las palabras. En Europa se suele identificar esta forma de capitalismo con el socialismo. Y en Estados Unidos, la corriente económica más progresista es el new liberalism (nuevo liberalismo) que fácilmente se puede confundir con lo que normalmente se llama neoliberalismo que es, prácticamente, lo opuesto. Por otra parte, términos como conservador (defensor de la tradición) y liberal (defensor de la modernidad), históricamente opuestos, hoy se identifican. Los sectores más conservadores son precisamente aquellos que defienden las políticas más liberales. Unas distinciones mucho más apropiadas son, desde luego, las que establece David Schweickart entre liberalismo clásico (Locke, Mill, A. Smith) hoy (Hayeck, Friedmann, Nozick) y liberalismo modemo, subdividido a su vez en liberalismo keynesiano (Keynes, P. Samuelson) hoy (J. Rawls) y liberalismo postkeynesiano, con una vertiente temprana (Galbraith) y una vertiente reciente, la más cercana al socialismo, a la que se suele llamar nuevo liberalismo (Thurow, P. Tsongas). David Schweickart compara las ventajas y desventajas de su modelo socialista con cada uno de estos modelos capitalistas, pero esto sobrepasa con creces las dimensiones de este comentario. 
por la importancia que ha adquirido en este último siglo, se ha convertido para muchos en sinónimo de socialismo a secas, y que consiste en que todos los medios de producción, los precios del mercado y los salarios son controlados por el Estado, y el socialismo autogestionario o democracia económica que nos propone, entre otros, David Schweickart².

La primera característica de la democracia económica consiste en la autogestión de las empresas por parte de los trabajadores, en democratizar las empresas de manera que cada trabajador tenga un voto y pueda elegir sus gerentes, dirigir su empresa, organizar el centro de trabajo, exigir disciplina, renovar las técnicas de producción y establecer las cuotas de producción. En este modelo socialista, el colectivo se reparte los beneficios, según un criterio establecido democrálicamente y elimina la plusvalía (explotación) y el trabajo como mercancía (enajenación) desde el momento en que el trabajador comparte el poder, los beneficios y los riesgos de la empresa. David Schweickart sugiere un régimen democrático empresarial que sea un punto medio entre el elitismo tecnocrático japonés y la ineficiencia que suele provocar una democracia excesiva. La segunda característica consiste en la persistencia de los mecanismos del mercado para establecer los precios y el valor de los salarios, evitando con ello la ineficacia de la burocracia, la irracionalidad de la sobrecentralización y la acumulación de poder en una élite, y la tercera característica sería el control social de la inversión para frenar la anarquía de la producción capitalista. Los fondos de inversión se generan por impuestos sobre los bienes de las empresas (máquinas, instalaciones, etc.) y se elimina todo tipo de interés por el dinero. Dichos fondos se invierten de acuerdo a una planificación del mercado, decidida por parlamentos democráticos. La democracia económica no pretende ser ni una copia, ni una síntesis de diversos modelos existentes, pero el autor tiene muy en cuenta un alto número de evidencias empíricas; los modos de organización del trabajo en distintos tipos de cooperativas, las lecciones del fracaso y de los éxitos del socialismo de Estado, las empresas relativamente autogestionarias de Yugoslavia, que tuvieron el crecimiento más alto del mundo desde 1952 hasta 1960, la economía japonesa con la intervención estatal a larga escala, la seguridad en el puesto de trabajo y las cuotas de participación del obrero, las cooperativas Mondragón de España con más de 180 empresas y un banco controlado democráticamente, y las reformas de la economía china, que no parecen encaminarse a ninguno de los modelos capitalistas conocidos.

Después de una prolija exposición de su sistema, pasa a compararlo, señalando pros y contras, con los demás. Pronto nos hace ver que la defensa no comparativa, per se del capitalismo, es más bien endeble. Nozick, Hayeck y Friedman, abanderados del capitalismo puro, argumentan que a pesar de las herencias, los regalos por razones arbitrarias y la caridad, el sistema capitalista es el que más se puede acercar a la distribución de bienes materiales, de acuerdo con los méritos de la actividad de cada uno. Pero mientras coordinar trabajo y capital y trabajar son actividades productivas, proveer capital, es decir, permitir usarlo, poner dinero en el banco, comprar acciones, invertir en un mercado de valores $y$, en general, las actividades centrales del juego capitalista, no lo son. La recompensa para aquellos que han arriesgado su dinero en cuentas bancarias, acciones y bonos del Estado es posible - y esta sigue siendo la verdad irrebatible del marxismo-, únicamente porque aquellos que producen cosas y servicios reciben menos por su contribución productiva. Uno solamente puede recibir algo por nada cuando alguien más recibe nada por algo. Si la distribución capitalista fuera hecha de acuerdo con el 'canon de contribución productiva, como algunos de sus defensores pretenden, entonces muchos capitalistas no recibirían nada. Del mismo modo, no se puede apelar a la retribución del sacrificio para justificar los pingües beneficios de los "proveedores de capital", porque muchos de los que se benefician del sistema no se sacrifican, ni a la retribución del riesgo, porque en el juego de inversión capitalista, a diferencia de la ruleta, no todos tienen los mismos números de ganar o perder. El gran inversor, por la mayor capacidad de diversificación de sus inversiones y su acceso a la información, tiene

2. También aquí hay muchos más tipos de socialismo: los sistemas de economía mixta, el socialismo participativo sin mercado, el socialismo tecnocrático de mercado, donde se nacionalizan las empresas, se mantiene el mercado y las ganancias son distribuidas por el Estado. Todos estos tipos son descritos y comparados por David Schweickart con el socialismo autogestionario. 
menos posibilidades de perder que el pequeño inversor, y la mayoría no puede ni tan siquiera jugar porque no tiene nada para invertir.

Sin embargo, la defensa más fuerte del capitalismo es la argumentación comparativa. Se procede señalando las ventajas y desventajas de los diversos sistemas económicos, y se concluye mostrando, como en un último balance, que el capitalismo, con todas sus injusticias, es el menos malo. Aquí es donde la argumentación de David Schweickart es más potente, llegándonos a persuadir de que la democracia económica se implementara, con todos sus inconvenientes, sería más eficiente, justa, democrática y ecológica que cualquier modelo de capitalismo posible. Por eficiencia entiende lo que los mismos teóricos del capitalismo puro han definido como tal: aquel estado de cosas en que la disminución del bienestar debido a una desviación de precios provocado por los monopolios y la ausencia de una competencia perfecta es mínima, en que el desempleo es inexistente y estructura interna de la empresa es óptima (motivación, dirección, rendimiento, etcétera). Es evidente que el capitalismo real se encuentra a leguas de distancia de todo esto, pero aun en un capitalismo ideal, donde los empresarios no conspiraran para fijar los precios o evitar que otras compañías entraran en su campo, no se alcanzaría la eficiencia de la democracia económica. Veámoslo con algún detalle.

En primer lugar, los defensores del capitalismo nos dicen que no hay ningún otro mecanismo mejor que el mercado libre para establecer el verdadero precio de las cosas, disciplinar la mano de obra, localizar al trabajador allí donde es más conveniente, minimizar los gastos materiales y favorecer el uso de tecnología apropiada para la producción. Pero el socialismo autogestionario, o democracia económica, no se opone al mercado, sino a la falta de libertad y democracia de este mercado. El mercado, pensado como una especie de foro democrático por los teóricos del sistema capitalista, es, en realidad, una democracia restringida, puesto que en el mercado cada persona no es un voto, sino cada dólar. Si uno no tiene un dólar, tampoco tiene voto. Los votos no sólo no están correctamente distribuidos, sino que 200 personas en el mundo tienen tantos votos en el mercado mundial como los 2,000 millones de personas más pobres. La democracia económica, al democratizar y "liberalizar" realmente el mercado, puede hacer que este instrumento sea tan eficiente como sus apologistas pretenden que es.

En segundo lugar, la democracia económica corrige una de las principales ineficiencias del capitalismo: los esfuerzos y el capital que literalmente se tira a la basura con el fin de favorecer a toda costa las ventas. En un mundo donde la mayoría tiene tan poco y hay tanta miseria y degradación ambiental no deja de ser chocante que se dedique tanto tiempo, inteligencia y capacidades a persuadir a aquellos que ya consumen demasiado a que consuman más, y que los ciudadanos de los países ricos estén tan obsesionados en competir. Es sencillamente insólito que tantos estén empleados en mejorar lo que no es mejorable, cuando el planeta tiene tantas necesidades. En Estados Unidos, el dinero gastado anualmente en marketing supera el gasto de gasolina, aceite y carbón e iguala a lo que se gasta en defensa: 300 billones de dólares anuales. En la democracia económica hay mucha menor tendencia a la venta irracional, y se incentiva y promueve justo lo contrario del capitalismo: que el trabajador quiera consumir menos y gozar de más tiempo libre. El ocio es la altemativa al aumento absurdo de las ventas. Si uno no conociera mínimamente los entresijos del sistema sería incomprensible que los políticos hablen tanto del desempleo, de la necesidad de controlar el gasto público e, incluso, de la protección del medio ambiente y la reducción de los gastos militares, sin apenas referirse al mayor despilfarro de nuestro tiempo: la publicidad.

En tercer lugar, diversos teóricos de la gestión empresarial consideran que la participación de los obreros en los objetivos, los beneficios y la organización de la empresa es la clave para mantener hoy su competitividad; sin embargo, afirman que no se debe llegar al extremo de la democratización de la empresa por la incapacidad del obrero o la ineficiencia de los mecanismos democráticos. Otra paradoja del capitalismo consiste en considerar a la gente capaz para elegir a representantes que pueden decidir impuestos, arbitrar leyes y declarar guerras, pero incapaz para elegir a sus jefes en el trabajo y tomar decisiones en aquello que más conocen: su propia empresa. Aquí, la democracia económica no hace más que derivar las conclusiones que se siguen de las teorías contemporáneas de gestión empresarial. La democracia económica, desde el momento que se introduce en el lugar del trabajo, permite a los problemas salir a la superfi- 
cie, ser debatidos y resueltos antes que lleguen a un punto crítico. Así, la posible pérdida de tiempo en la toma de decisiones democráticas, en realidad es ganancia para la empresa y siempre se puede mejorar con sistemas democráticos más ágiles. De hecho, la democracia económica fortalece y defiende la misma democracia política y la recobra de su languidecimiento pues, por más que se asocie el capitalismo con la democracia política, éste no le da más que un valor instrumental y está muy interesado en mantenerla bajo mínimos. De lo que se trata en la democracia capitalista es simplemente del derecho a cambiar los altos funcionarios del gobierno sin que los ciudadanos estén razonablemente informados sobre las cuestiones a ser decididas en los procesos políticos. Además, los bancos y las corporaciones financieras se encargan de modular el clima social y político en las campañas electorales, mientras que los ricos están magníficamente organizados para defender sus intereses, acceder fácilmente a las altas jerarquías del gobierno y estar desproporcionadamente representados en ellas.

En cuarto lugar, mientras aún en el capitalismo ideal es imposible el pleno empleo, en la democracia económica no, y al menos ésta tiene mayor capacidad para generarlo. Una empresa autogestionaria puede contratar siempre más trabajadores, reduciendo las horas de trabajo, mientras que la empresa privada no tiene ninguna tendencia intrínseca a reducir drásticamente la jornada laboral si no aumentan con ello sus beneficios. En el capitalismo hay demasiada tolerancia para un asunto tan grave y con unos efectos sociales, psicológicos y políticos tan perniciosos como los que produce el desempleo. Normalmente se arguye que el pleno empleo favorece la burocracia y limita la iniciativa, la creatividad y la disciplina de la mano de obra, pero en la democracia económica, la gente gana dependiendo de la competitividad de la empresa. No desaparecen ni la competitividad empresarial, ni los despidos de trabajadores ineficientes o irresponsables, ni el cierre de las empresas cuando éstas fracasan.

En quinto lugar, se asegura que las empresas autogestionarias no pueden arriesgar tanto como los inversores capitalistas, ni diversificar del mismo modo sus inversiones; que el capital acumulado tenderá a evitar riesgos y, en consecuencia, habrá menos innovaciones e iniciativas. Sin embargo, en la democracia económica los riesgos son los mismos que en la economía capitalista, sólo que se encuentran más socializados y el capitalista está liberado de este sufrimiento. Los obreros con iniciativa reciben subvenciones de los bancos y se comprometen a pagar un impuesto por los bienes materiales adquiridos con la subvención, pero no a repararla. Con más razón se afirma que en la democracia económica no habrá el incentivo para un gran negocio y que la excitación y la motivación para empezar algo nuevo no bastan para estimular la innovación, pero, si esto fuera así, se podría permitir que las empresas fueran manejadas, al principio, como empresas capitalistas, dejando todo el provecho a los iniciadores, y en un cierto punto, obligarlas a democratizarse. La flexibilidad aquí, como en cualquier fórmula, es decisiva. Por otro lado, en la democracia económica es más fácil trabajar con sentido en la medida en que todos participen en el desarrollo de la empresa. Las mismas teorías de gestión empresarial reconocen que hoy puede ser más decisivo para la competitividad de la empresa el trabajo con sentido que el mero estímulo económico. Incluso un autor liberal como Stuart Mill reconoce que de trabajar ciegamente y para el provecho de otros no resulta nunca un estado satisfactorio.

En sexto lugar, la democracia económica también parece mucho más capaz de hacer frente a los retos ecológicos, pues los trabajadores controlan la tecnología, tienden más a vivir cerca de la empresa y no sienten la necesidad imperiosa de crecer. Según parece, una vez las necesidades básicas están cubiertas, el aumento en el nivel del consumo no implica mayor felicidad. Incluso podría ser que el exceso de consumo y de aparatos fuera parte del problema de aburrimiento, soledad y vacío que padecen las sociedades ricas. ¿Acaso no es mejor tener tiempo para cultivar amigos, desarrollar habilidades, leer, visitar lugares, que aumentar el consumo? En el capitalismo es imposible llegar a un estado de crecimiento estacionario, porque la clase adinerada, que puede vivir sin trabajar, encuentra su única legitimidad en el crecimiento. Si en algún momento los consumidores no continuaran comprando más, si estuvieran demasiado satisfechos, provocarían una grave crisis. En la medida en que en la democracia económica no hay una clase económica cuya única justificación sea el crecimiento, es más susceptible llegar a un estado de equilibrio.

Por último, la democracia económica favorece más la justicia y la igualdad que el capitalismo. 
Téngase en cuenta que en Estados Unidos, la diferencia media entre el salario de un ejecutivo y el salario medio de la nación es, en la actualidad, de 85 veces, mientras que en las cooperativas Mondragón es de seis. El capitalismo tiende a generar desigualdades masivas en el mundo, bolsas de miseria y delincuencia cada vez mayores en los países pobres e, incluso, en el mejor de los países capitalistas, las grandes fortunas de algunos corrompen el carácter de la mayoría, provocando envidias y hasta una absoluta falta de respeto y de confianza en sí mismos cuando no se tiene éxito económico. En la democracia económica subsiste la desigualdad, pero no se permiten los extremos, ya que en ella es imposible que haya superricos ni ingresos que no sean ganados trabajando, es decir, con algún tipo de labor productiva, ya sea física o mental.

Ahora bien, si las empresas autogestionarias son más eficientes que las empresas capitalistas, ¿por qué no han llegado a ser la forma predominante de organización? Diversas razones apuntan a ello. Primero, se da la circunstancia que cuando las cooperativas compiten en una economía capitalista, el acceso a las finanzas, la propaganda y las estructuras de apoyo no es igual. Incluso, si las cooperativas son preferidas por la mayoría y son más productivas, un mercado dominado inicialmente por el capitalismo no las selecciona. Segundo, no suelen tener la misma motivación para expandirse que las empresas capitalistas y en un mercado capitalista sus mejores trabajadores tienden a irse a empresas privadas. Tercero, históricamente, los sindicatos obreros han considerado a las cooperativas como una traición a la clase y a la batalla por el socialismo, y no siempre las cooperativas se han insertado en un proyecto social alternativo. Por último, hay que contar con la hostilidad ideológica. De alguna manera, aunque ya no haya comunismo, sigue el anticomunismo. Por más democrático que sea un movimiento, si amenaza la propiedad privada de los medios de producción, será combatido por las fuerzas del capital.

Sin embargo, quedarnos con la satisfacción intelectual de saber por qué la democracia económica con todas sus virtudes, no se da todavía, y por qué los defensores del capitalismo están equivocados, sabe a bien poco. Con todo lo decisivo que pueda ser saber que no existe ninguna justificación ética o económica para el capitalismo y que si éste persiste no es porque no haya una alternativa socialista más viable y deseable, sino porque aquellos que más se aprovechan tienen mucho poder para evitar la emergencia de cualquier orden nuevo, no deja de parecer un ejercicio algo estéril. Pero no hay ninguna inevitabilidad histórica y el mismo planteamiento de un orden alternativo viable puede ser un magnífico detonante para elaborar estrategias y aglutinar fuerzas capaces de empujarlo. De hecho, como apunta David Schweickart, sería posible una transición abrupta del capitalismo a la democracia económica mediante una acción gubernamental que arbitrara cuatro leyes: (1) todo ingreso basado en intereses y acciones es nulo y las compañías dejarán de pagar dividendos; (2) todas las empresas que empleen a más de un determinado número de trabajadores pasan a ser controladas democráticamente por éstos. Cada trabajador tendrá un voto. La única restricción es que el valor del capital de la empresa deberá permanecer intacto; (3) los bancos pertenecerán a la comunidad donde están localizados. Serán pagados mediante impuestos; (4) toda empresa debe pagar un impuesto a la comunidad por el uso de bienes. Pero, en la actualidad, este escenario es más bien improbable aunque, por ejemplo, la reforma de Meidner, impulsada en 1970, en Suecia, por la que el 20 por ciento de la tasa de beneficio de las empresas de más de 500 trabajadores debía ponerse en manos del sindicato anualmente, suponía que en 20 años, los trabajadores controlarían la mayoría de las industrias.

Pese a todo, se pueden realizar múltiples acciones que pugnen para que lo que hoy se encuentra en estado embrionario adquiera perfiles más definidos. David Schweickart destaca, en primer lugar, la promoción de empresas democráticas en todo el mundo. Si en el primer mundo la principal dificultad es la competencia, en el tercero suele ser la falta de formación y hábitos de responsabilidad; pero en todas partes hay experiencias cooperativas exitosas. En segundo lugar, la presión para que las empresas capitalistas sean más participativas, reconozcan el derecho a inspeccionar los beneficios de la empresa y a tener representantes en la junta de directores, y favorezcan el trabajo en equipo, la calidad del trabajo y la reducción del horario laboral. Del mismo modo que los movimientos feministas han denunciado que no se puede ser revolucionario en la calle y machista en la casa, y que ha sido un vicio bastante común de la izquierda defender las causas justas fuera y tolerar la injusticia en casa, la democracia económica nos 
exige acercar nuestros hábitos cotidianos en el trabajo a la alternativa defendida, e integrar la lucha política en el día a día. Y en tercer lugar, la necesidad de revitalizar el movimiento sindical democrático, porque aunque históricamente éste se ha opuesto a los programas cooperativos considerando que rompían la solidaridad de clase, sin una gran fuerza sindical es imposible avanzar hacia la democracia económica. Es palmario que todo aquello que toque las raíces mismas del capita- lismo tendrá que enfrentarse con poderosos intereses, y que un movimiento recio, comprometido e inteligente de ámbito mundial, es indispensable para desafiar a una clase dominante tan potente como la actual, pero que el éxito no sea fácil no significa que no se pueda triunfar. Hay fuerzas identificables que pueden empujar estos cambios, alternativas viables y esperanzas razonables.

\section{Jordi Corominas}

